\title{
PowerPoint presentations in the classroom: Re-evaluating the genre
}

\author{
Larissa D'Angelo \\ larissa.dangelo@unibg.it \\ Department of Foreign Languages, Literatures and Cultures \\ University of Bergamo, Italy
}

\begin{abstract}
Since the late 1990s, Microsoft PowerPoint has become the expected presentation genre. However, several studies have demonstrated its many faults, such as the pre-formatted construction of discourse leading to the abuse of bullet point presentations, the limited format and size of slides that support minimum content and the ever-present risk of overwhelming viewers with too much text or data (Alley 2003, 2004, Robertshaw 2004, Gottlieb 1985, Keller 2003, Tufte 2003). Taking into consideration how the linguistic and visual elements, as well as the design and text organizations found in PowerPoint presentations have evolved in the last 20 years, the present paper analyses the negative effects that the default slide structure provided by Microsoft PPT, consisting of topic-subtopics and bullet points, has on the audience. The paper will then demonstrate the positive learning effects that the assertion evidence structure has on readers. The different retaining degree of three groups of undergraduate students are tested, after having exposed them to PPTs applying phrase headlines, phrase headlines and images or the assertion evidence structure.
\end{abstract}

Keywords: PPT, PowerPoint, PowerPoint presentation, multimodality, multimodal genre, multimedia design

\section{INTRODUCTION}

In today's academic world, PowerPoint presentations have become increasingly common not only in the hard sciences but also in the humanities, showing how the fast-paced, visually attractive data-driven presentations typical of marketing and business have invaded even the most traditional settings. As Tufte (2009) confirms, "slideware - computer programs for presentations - is everywhere: in business settings, in government bureaucracies, even in our schools and universities, where several hundred million copies of Microsoft PowerPoint are generating trillions of slides each year." Indeed, if the conventional method of presenting research results at conferences, 
workshops and even university lessons was to stand in front of an audience reading a paper, scribbling on lucid or writing formulas on a blackboard, today lectures have been enriched with images, colour and sometimes even music and videos thanks to new, enhanced software. As Myers (2003: 3) recognises,

[...] anyone who walks around a university campus today will soon be aware that academic discourse is not just about words. There are colour-illustrated textbooks, videos, and interactive whiteboards boards in teaching sciences, materials and actions in labs, lectures and demonstrations, PowerPoint presentations in university lectures, web pages as support for teaching and publicity, and music signalling the scientific in television documentaries.

PowerPoint as a multimedia tool is used not within University walls but also by primary school teachers teaching K-12 grades (Martin and Carr, 2015). The software is the most utilized tool and is used daily to introduce new topics, explain concepts and presumably enhance lessons by integrating multimodal exercises (2015: 10-11). Among a number of multimedia software available, enabling them to create multimodal material for K-12 students, teachers still choose first and foremost PowerPoint, followed by Vimeo, Youtube, Camtasia, Animoto, Prezi and xtranormal (2015: 8). Why has this surplus of multimodal instruments invaded the academic world so strongly in the past few years? As Myers (2003: 3) states, science has always been multi-modal; historians have shown that it is our own textual bias that cuts out the elements of the visual and the performed from past scientific practice (Gross et al. 2002). But it could be that new technologies make it easier to carry non-verbal elements from medium to medium, and easier to interweave different modes.

The effects of technology on academic discourse are numerous and sometimes insidious, changing what were once 'traditional genres' such as the research article and the lecture into multimodal genres, requiring new preparation and delivery skills and a new approach to genre analysis. Myers (2000: 184) offers a particularly rich and illuminating discussion of the intersection of technology and genre in which he discusses the effects of PowerPoint on his own lecture preparation, delivery, and 30 
reception. After dealing with the more obvious consequences, such as the 'bulletization' of information, he goes on to write:

[...] the written text, produced by the machine, has become the star; I am reduced to an unseen voiceover of my own lectures. That may not matter in a business setting, where different people from sales or personnel may be called upon to speak the same words. But for a university lecturer, it marks a shift in what Goffman (1981) called footing; that is, I am seen as the animator rather than the source of the utterance. Instead of my speaking with the aid of some visual device, the text is speaking with my aid.

Swales (2004: 7) reinforces Myers' account of technological impact by stating that certain multimodal genres, such as the PowerPoint, inevitably blur the boundaries between the academic and the commercial, and between the written and the visual.

Along the same lines, Rowley-Jolivet (2001) observes that the frequent use of photographs in Conference Presentations (hereinafter CP) reinforces the sense that these presentations often deal with early-stage, breaking-news research. Given the CP time pressures, the idea that "a picture is worth a thousand words" has clearly come to the fore (Swales 2004: 199). For this reason, visual presentation and graphics in conference PowerPoint presentations and handouts have become vital to outline a piece of work in a form that is easily assimilated and stimulates interest and discussion (Matthews 1990, Tufte 1990).

How has the genre of oral presentations evolved through the years and how is it that PowerPoint invaded university classrooms and conference venues? More importantly, how does PowerPoint's traditional format modify academic discourse? Long before today's presentation programs, such as Microsoft PowerPoint, OpenOffice.org, Impress or Apple iWork Keynote, presentations at companies such as IBM and in the military used bullet lists shown by overhead projectors. However, the format has become omnipresent as PowerPoint, which was created in 1984 and later acquired by Microsoft, spread around the world. This spoken/written genre has evolved together with 
technology and its popularity has raised several debates concerning its common practice use at conferences (Keller 2004, Parker 2001, Schwartz 2003).

Visual aids and computer presentations can enhance speaker credibility and persuasion, increase audience interest, focus audience attention, and aid retention of key points/content, although the exact opposite is also true when visual aids and computer presentations are used poorly by a speaker (Stoner 2009). In fact, presentation programs may help speakers organize their talks, but what is convenient for the speaker might be detrimental to both content and audience. The typical PowerPoint style suggested by the program itself and the ready-made templates available to Microsoft users routinely disrupt, dominate, and trivialize content, elevating format over content and betraying an attitude of commercialism (Tufte 2009).

Since the 1980s, Gottlieb (1984) and others (Alley 2003, Atkinson 2005, Doumont 2005, Gaudelli et al. 2009, Keedy 1982) have rejected phrase headlines, responsible for unclear main assertions and lack of connections in the evidence, and have advocated the assertion-evidence structure, which features a sentence-assertion headline supported by visual evidence (see Figure 1).

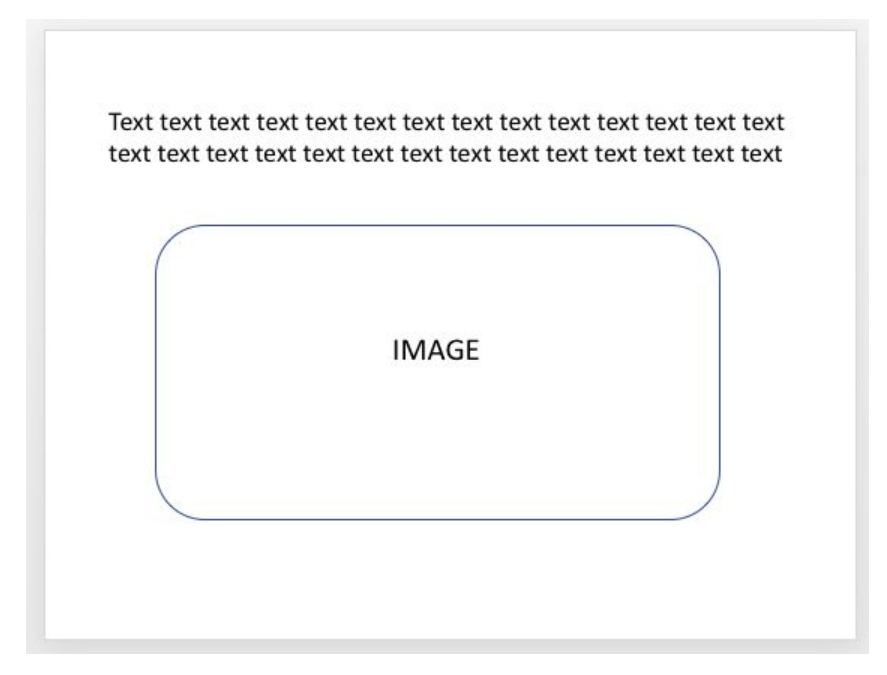

Figure 1. PPT slide employing the assertion-evidence structure. 
How does the assertion-evidence structure work? When a presentation slide appears before the audience, the audience immediately turns to it and tries to decipher its contents and purpose. The assertion-evidence structure helps the audience quickly understand and retain the contents of a slide by providing a sentence headline, which orients the audience to the purpose of the slide; the audience can then turn its attention back to the presenter. Once the presenter has made clear what the main message of the slide is, the presenter should support that assertion primarily with images and with words where needed. The reasoning for this guideline is that images, if well-conceived, can communicate information much more quickly to the audience than blocks of text can.

Using a sentence headline is not the norm in scientific presentations (Alley and Robertshaw 2004). In fact, because thousands of presentations typically use phrase headlines (or no headlines at all), the assertion-evidence structure goes against what is most often seen and recommended. Phrase headlines in presentation slides in fact, should be avoided because they seem to reduce the personal connections between the presenter and audience, thus disturbing the flow of information and reducing the persuasive force of the message.

Because presentation slides reduce the personal connections between the presenter and audience, presenters have to be critical thinkers about the reader-oriented strategies employed and, most of all, when this academic genre is appropriate and when it is not (Alley 2003).

Taking into consideration how the linguistic and visual elements, as well as the design and text organizations found in PowerPoint presentations have evolved in the last 20 years, the present paper analyses the negative effects that the default slide structure provided by Microsoft PPT, consisting of topic-subtopics and bullet points, has on the audience. On the other hand, the paper will demonstrate the positive learning effects that the assertion evidence structure has on readers. More specifically, the different retaining degree of three groups of undergraduate students will be tested, after having 
exposed them to PPTs applying phrase headlines, phrase headlines and images or the assertion evidence structure.

\section{METHODOLOGY}

Two main methods for presenting data will be taken into consideration and analysed hereafter: on the one hand the standard method for presenting information through the projection of data in bullet-style and/or graphical formats i.e. phrase headlines, on the other hand the assertion-evidence structure, which is gradually gaining acceptance in the hard sciences.

To ascertain the positive or negative effects of these two different types of PPT formats on the audience and on the university student population in particular, three groups of undergraduate students attending the University of Bergamo between October 2015 and December 2017 have been selected and exposed to the same subject matter, which was however presented in different forms (see Table 1). The first group, counting 58 students, served as the control group and was exposed to a 30-hour module, entitled 'The language of written advertisements in English', which took place in the first semester of the academic year 2015/16 and was addressed to first year students enrolled in the Intercultural Communication for Co-operation and Business undergraduate degree programme. The control group was exposed to lessons utilizing PPT presentations, which employed "bullet style" phrases, interspersed with graphs and chunks of text. The second group counted 45 students enrolled in the same undergraduate degree programme and exposed to the same 30-hour module, which took place the following academic year, although in this case, relevant illustrations were added to text-only presentation materials. The third group counted 62 students, enrolled in the same degree programme as the previous two groups of students and attending the same course, although the 30-hour module took place, in this case, in the first semester of the academic year 2017/18. The latter batch of students was exposed to carefully re-designed slides organized according to the assertion-evidence structure (Figure 1).

34

Language Value 10 (1), 29-44 http://www.e-revistes.uji.es/languagevalue 
Table 1. Tests conducted and group characteristics

\begin{tabular}{|c|c|c|c|c|l|}
\hline Group & Semester & $\begin{array}{c}\text { Academic } \\
\text { Year }\end{array}$ & $\begin{array}{c}\text { Number } \\
\text { of } \\
\text { students }\end{array}$ & $\begin{array}{c}\text { Number } \\
\text { of course } \\
\text { hours }\end{array}$ & \multicolumn{1}{|c|}{ Material used } \\
\hline 1 & $1^{\text {st }}$ & $2015 / 2016$ & 58 & 30 & $\begin{array}{l}\text { Topic-subtopic slide design, "bullet style" } \\
\text { phrases with graphs and chunks of text }\end{array}$ \\
\hline 2 & $2^{\text {nd }}$ & $2016 / 2017$ & 45 & 30 & $\begin{array}{l}\text { Topic-subtopic slide design, "bullet style" } \\
\text { phrases with graphs, chunks of text and } \\
\text { illustrations }\end{array}$ \\
\hline 3 & $1^{\text {st }}$ & $2017 / 2018$ & 62 & 30 & $\begin{array}{l}\text { PPT slides following } \\
\text { assertion-evidence structure }\end{array}$ \\
\hline
\end{tabular}

All the students selected for the research had an attendance rate of at least $90 \%$, had all studied English for at least five years before the beginning of the course, and reached a certified B1 level of English. All students were administered the same multiple-choice test on the last day of the course and were given 30 minutes to complete the task. Students were administered the test without notice so as to measure the retention of the material to which they had been exposed over the previous 3 months, without preparation.

The test featured 20 questions focusing on the content of the course and 5 different answers were provided for each question. The results obtained by the second and third group were compared with the results obtained by the control group and the relevant statistics were drawn, demonstrating the positive effects of adding relevant images to PPT slides and using the assertion-evidence structure.

\section{RESULTS}

The investigation described in this section relies heavily on the generative theory of multimedia design (Mayer 2001). This theory is based on the view of learning as knowledge construction, the idea that learner's actively build mental representations 
based on what is presented and what they already know. It therefore advocates that materials that facilitate selection, organization, and integration of to-be-learned information are of benefit in designed instruction.

The following hypotheses have been tested on three groups of non-native undergraduate students, following a 30-hour module in English:

1. By simply adding relevant illustrations to text-only presentation materials, retention increases;

2. People comprehend and retain better without extraneous information (learning material must be simplified, removing everything that isn't directly related to the discussion).

As Table 2 shows, the first group of students, which served as the control group for the research, scored an average of $68 \%$ on the final test. These students were exposed to learning material which consisted mainly in PPT slides employing "bullet style" phrases, interspersed with graphs and chunks of text. A limited number of images were shown in PPT slides and numerous references were instead made to the textbook adopted for the course.

Table 2. Retention increase with different PPT layouts

\begin{tabular}{|l|l|l|l|}
\hline Group & Material provided & $\begin{array}{l}\text { Average score on } \\
\text { test (\%) }\end{array}$ & $\begin{array}{l}\text { Retention } \\
\text { increase (\%) }\end{array}$ \\
\hline 1 (control group) & Mainly text-only PPT slides & $68 \%$ & - \\
\hline 2 & PPT slides with text and images & $87 \%$ & $+19 \%$ \\
\hline 3 & $\begin{array}{l}\text { PPT slides displaying the } \\
\text { assertion-evidence structure. }\end{array}$ & $94 \%$ & $+26 \%$ \\
\hline
\end{tabular}

The second group of students was exposed to the same PPT slides, to which relevant images were added. Unlike the learning material provided to the previous group, in this case, almost every slide included at least one picture relevant to the subject matter. At

36

Language Value 10 (1), 29-44 http://www.e-revistes.uji.es/languagevalue 
the end of the course these students scored $87 \%$ on the test given, demonstrating that just by adding relevant illustrations to text-only presentations, retention increased by $19 \%$.

The third group of students were exposed to carefully re-designed PPT slides, employing the assertion evidence structure. Learning material was simplified, removing everything that was not directly related to the discussion and a sentence headline was used, followed by a clear picture or simplified graph, reinforcing and/or complementing the information given in the headline. Sentence headlines were no longer than two lines so as to avoid heavy chunks of text and the total number of slides provided was less numerous, so that in certain instances information was conveyed only orally and relied on the rhetorical capabilities of the presenter. The students belonging to this group scored an average of $94 \%$ on the final test, showing that the use of the assertion-evidence structure increased retention by $26 \%$.

This last finding provides a powerful incentive not only to re-design and re-think the layout of university course materials, but also to implement the assertion-evidence principle whenever a transfer of knowledge is required as in academic meetings, conferences and workshops. It is also a strong incentive to avoid including graphics or multimedia effects simply for the sake of including them and to incorporate only the graphics that closely relate to the content, removing all extraneous, distracting details (Sommers 2008).

\section{DISCUSSING THE ASSERTION-EVIDENCE STRUCTURE: SWIMMING AGAINST THE CURRENT}

As introduced in the previous sections, since the late 1990s, Microsoft PowerPoint has become the expected presentation genre, because it is the most commonly pre-installed software in PCs and Macs alike. The software developed from a culture of slides within business, government, and military organizations, with the latter particularly fond of bullet phrases in documentation, long before the introduction of electronic presentations. In particular, PowerPoint was introduced when the form of 
communication began requiring interaction in different forms both horizontally and vertically within an organization (Pece 2005).

In corporate history, DuPont has been one of the first users of charts and graphs to be viewed in a special chart-viewing room. This practice was widely copied and what was “uniquely DuPont" (Orlikowski and Yates 1994) - use of graphs as visual aids became more widespread. By the second half of the 20th century, visual aids became the norm and the pre-processed 'bullet style' presentation of information became the standard rhetorical construction employed in academic and non-academic settings.

The shift from carefully crafted lucid presentations and expensive $35 \mathrm{~mm}$ slides to ready-to-use and widely accessible PPT slides has revolutionized and standardised rhetoric, deconstructing the art of oratory within University walls (Keller 2004, Parker 2001, Myers 2000, Tufte 2003). Its design forces users to follow a pre-formatted construction of discourse, encouraging an abuse of bullet point presentations; the format and size of slides do not support much content and tables as well as graphs, if presented through a PPT slide, hold very little information and the risk of overwhelming viewers with too much text or data is ever-present (Alley and Robertshaw 2004, Keller 2003, Tufte 2003). If these negative aspects were not enough, Tufte (2003) has correctly underlined that the biggest fault of the software is its tendency to "dilute thought" (2003:6), encouraging a "generic, superficial, simplistic thinking" (2003: 5). Although it simplifies the presenter's task of delivering oral discourse because of its bullet point style, its design limits and slows down the flow of information; simply reinforcing what is being said, thus rendering this tool inadequate to for complex, non-linear issues.

Another fault lies in the quick loss of audience attention because listeners are led to shift their attention from the speaker to the screen, quickly tuning out the presenter and concentrating solely on the text. If slides utilize fonts that are not easy to read or they overwhelm readers with too much text, the ultimate outcome is that viewers, who have stopped listening, eventually stop reading too, losing all interest in the presentation because the material shown was not able to trigger an emotional response.

38

Language Value 10 (1), 29-44 http://www.e-revistes.uji.es/languagevalue 
There are however recent studies that present and promote alternative uses of the software, so that it can be correctly utilized in an educational setting. For example, Lai, Tsai and Yu (2011) propose a Two-Layer display of information on screens to avoid overloading students with information. Kumar's (2013) study supports the position described above, indicating that students preferred PowerPoint over blackboard-based lectures, because the "inherent deficiency of each method is compensated by the other. While blackboard teaching is deficient in showing three dimensional diagrams, animated videos, and sounds; the same can be demonstrated using a PowerPoint presentation" (p. 240).

A solution to this dilemma is provided by the assertion-evidence structure utilized with group 3 in the present study. This structure proposes the use of full sentences instead of phrase sentences, which are typically fragments of phrases and do not help viewers comprehend immediately what is being shown in the slide and, most of all, do not favour retention of the subject matter. Alley and Robertshaw (2004) suggest placing the sentence headline in the upper-left corner of the slide, so that the audience sees the headline before anything else on the slide and to favour a quick retention, it should be no more than two lines long and justified left.

Several good reasons exist for using sentence headlines. One is that a sentence headline forces the presenter to come to rehearse and carefully select the assertions he or she is making (Alley 2003, 2004, Alley and Robertshaw 2004, Gottlieb 1985). The presenter is in a better position to select the best evidence to support those assertions because s/he has clearly established what the assertions of the presentation are when s/he wrote the easily-readable headlines to display on the slides. A second reason is that using sentence headlines makes the set of slides stand-alone better as a set of notes. For instance, if a slide simply had the headline "Results," it would not be nearly as helpful to the audience two weeks later when viewed as part of a set of notes. The 'Results' slide displaying a short headline, summarizing the main results is much more effective and useful once notes are re-read at home. 
A third reason for the value of sentence headlines is that presentations using sentence headlines tend to have significantly fewer slides (Alley and Robertshaw 2004), thus reducing the frenetic pace that weakens so many presentations. The reason for the reduction in the number of slides is that if the presenter cannot write a sentence for the slide that states its assertion, the design calls for the elimination of the slide (Alley 2003, Gottlieb 1985).

Once the presenter has established what the main concept of the slide is with the sentence headline, he or she supports that concept primarily with images and with words (where needed). Images, if well-conceived, can in fact communicate information much more quickly to the audience than blocks of text. If a block of text must be included in a slide, it should be no longer than two lines, including the headline, because audiences are much more likely to read blocks of text with one or two lines than longer blocks.

Because audiences are more likely to remember lists of twos, threes, and fours than lists of fives, sixes, or sevens, lists with more than four items should be avoided. Moreover, when a long list is presented, the audience sees the length, perhaps reads the first couple of items, and then tends to give up on the remaining items. When a long list must necessarily be included in a slide, presenters should then place only the four most important items from that list onto the slide and reserve the less important items for the speech (Alley and Robertshaw 2004).

Another useful technique that comes with the use of the assertion-evidence structure is to be generous in the use of white space, because it prevents a slide from seeming overcrowded (Hill 2004). White space not only allows the audience to separate the items in the slide's body, but also helps viewers find a logical order in which to view them.

Presenting something following a non-standard PPT layout requires a deep understanding of the subject matter as bullet point prompts are no longer available. Therefore, the assertion-evidence structure demands a much greater preparation by the presenter than a standard PPT presentation, besides the difficulty of applying changes to 
the typography and layout of slides. However, as the previous section demonstrated, the results of applying this new design are well worth the effort.

\section{CONCLUSIONS}

The present research has highlighted the potentialities of presentation software such as Microsoft PPT, as well as the numerous criticisms this genre has collected in recent years. PPT slides have become the standard format to present ideas and transfer knowledge not only in governmental and business settings, but within the academy as well.

In the humanities, PPT presentations have spread incredibly fast and have become the norm not only in classrooms but also in academic conferences and seminars. Viewers have come to expect (and respect) what has been defined as 'group wall reading', often not realizing that PPT slides are not always the best vehicle of information and can easily become a medium, which hinders communication instead of facilitating it.

There are numerous faults inherent to the software, such as the fact that very little information can be conveyed on each slide, limiting content to a series of bulleted lists and fragmented sentences. Microsoft automatically suggests a standard form of presentation, rich in special effects that can be visually appealing but also unnecessarily distracting. All these elements allow slides to dominate over the speaker and instead of being a means to enrich messages, slides become the message itself. The dominance of projected slides over the speaker often means that presenters forego an important opportunity to connect with the audience and in many cases the message is lost because of a lack of clarity, overwhelming information or simply a lack of interest.

Given the numerous drawbacks this software has, why should academics still use it? Because, as the results of this research have demonstrated, despite its potentially dangerous features, a PPT - if well used - can become a tremendously effective communication tool. Because of its multimodal nature, it is capable of combining text, but also images, graphs, movies and music. If slides are properly reorganized, redundant

41

Language Value 10 (1), 29-44 http://www.e-revistes.uji.es/languagevalue 
text and disturbing special effects eliminated, and images are added to complement the message, a PPT can become an excellent medium of communication.

In order to achieve this aim, a presenter must necessarily regain confidence in his/her oratorical skills, allowing slides to simply enrich and accompany what the presenter has to say, instead of dominating the presentation with redundant text, lists and graphs, which are bound to be read aloud, sadly distancing the presenter from the audience.

Making a transition from the now 'traditional' slide format, to a format such as the assertion evidence structure, is not an easy and requires substantial work, a deep knowledge of the subject matter and most of all, enough confidence to 'navigate solo', not using the software as a mere prompter but as an accompanying and enriching tool.

\section{REFERENCES}

Alley, M. 2003. The Craft of Scientific Presentations. New York: Springer-Verlag.

Alley, M. and Robertshaw, H. 2004. Rethinking the Design of Presentation Slides: The Importance of Writing Sentence Headlines. ASME IMECE (Anaheim, CA: ASME, 2004), paper 61827.

Atkinson, C. 2005. Beyond Bullet Points: How to Use Microsoft PowerPoint to Create Presentations That Inform, Motivate, and Inspire. Redmond, WA: Microsoft Press.

Atkinson, C. 2009. "The Problem with PowerPoint". BBC NEWS. 19 August 2009. http://news.bbc.co.uk/go/pr/fr//2/hi/uk_news/magazine/8207849.stm

Doumont, J. 2005. "The Cognitive Style of PowerPoint: Not All Slides Are Evil". Technical Communication, 52(1), 64-70. 
Gaudelli A., Alley M., Garner, J. and Zappe S. 2009. "Common use of PowerPoint versus the Assertion-Evidence Structure: A cognitive psychology perspective”. Technical Communication 56, 331-345.

Goffman, E. 1981. Forms of Talk. Oxford: Basil Blackwell.

Gordon, S. 2001. Really Bad PowerPoint (and how to avoid it). 25 August 2009. http://www.sethgodin.com/freeprize/reallybad-1.pdf

Gottlieb, L. 1984. "New-Breed Presentationists Sometimes Closely Collaborate on Presentations". Proceedings of the 1984 Professional Communication Society Conference of the IEEE. Atlantic City, NJ: IEEE.

Gottlieb, L. 1985. Well Organized Ideas Fight Audience Confusion. Livermore, CA: Lawrence Livermore National Laboratory.

Gross A., Harmon J. and Reidy M. 2002. Communicating Science: The Scientific Article from the 17th Century to the Present. Oxford: Oxford University Press.

Hill, J. 2004. “The Writing on the Wall”. Presentations (March 31, 2004).

Keedy, H. 1982. PRO Visuals Can Improve Your Presentations, Sixth Annual Practical Conference on Communication Proceedings (Knoxville, TN: University of Tennessee, October 22-23, 1982), 13-34.

Keller, J. Y. 2004. “Is PowerPoint the Devil?”. Chicago Tribune (23 January 2004).

Kumar, M. P. 2013. "Preferences of undergraduate medical students: Electronic and non-electronic teaching methods in pathology". International Journal of Research in Health Sciences, 1, 239-241.

Lai Y., Tsai H. and Yu P. (2011). "Screen-Capturing System with Two-Layer Display for PowerPoint Presentation to Enhance Classroom Education". Educational Technology \& Society, 14(3), 69-81.

Martin F., and Carr M. L. 2015. "An Exploratory Study on K-12 Teachers' Use of Technology and Multimedia in the Classroom". Journal of Educational Technology, 12(1), 7-14.

43

Language Value 10 (1), 29-44 http://www.e-revistes.uji.es/languagevalue 
Matthews, D. L. 1990. “The Scientific Poster: Guidelines for Effective Visual Communication". Technical Communication 37(3), 225-232.

Mayer, E. R. 2001. Multimedia Learning. Cambridge: Cambridge University Press.

Myers, G. 2000. "Powerpoints: Technology, Lectures, and Changing Genres". In Trosborg, A. (ed.) Analysing Professional Genres. Amsterdam: John Benjamins Publishing Company.

Myers, G. 2003. "Words, Pictures and Facts in Academic Discourse”. Iberica, 6, 3-13.

Orlikowski J. W., and Yates J. (Eds.), "Genre Repertoire: Examining the Structuring of Communicative Practices in Organizations". Administrative Science Quarterly, 39, 541-574.

Parker, I. 2001. “Absolute PowerPoint”. The New Yorker (28 May 2001).

Pece, S. G. 2005. The PowerPoint Society: The Influence of PowerPoint in the U.S. Government and Bureaucracy. MA Thesis, Virginia State University.

Rowley-Jolivet, E. 2002. "Visual Discourse in Scientific Conference Papers: A Genre-based Study". English for Specific Purposes, 21,19-40.

Schwartz, J. 2003. "The Level of Discourse Continues to Slide". The New York Times (28 September 2003).

Sommers, A. 2008. Tips for Designing Persuasive Slide Presentations. 07 June 2018. http://www.businessperform.com/articles/training-practice/slide-presentation-des ign-tips.html

Stoner, G. M. 2009. Effectively Communicating with Visual Aids. 10 July 2018. mattstoner.net/presentations/effective_visuals.pdf

Swales, J. 2004. Research Genres. Explorations and Applications. Cambridge: Cambridge University Press.

Tufte, E. 1990. Envisioning Information. Cheshire: Graphics Press LLC.

Tufte, E. 2003. The Cognitive Style of Power Point. Cheshire: Graphics Press LLC.

44

Language Value 10 (1), 29-44 $\quad$ http://www.e-revistes.uji.es/languagevalue 
Tufte, E. 2009. “Power Point is Evil”. Wired (November 2009). 
Received: 6 May 2018

Accepted: 23 July 2018

Cite this article as:

D'Angelo, Larissa 2018. "PowerPoint Presentations in the Classroom: Re-Evaluating the Genre". Language Value 10 (1), 29-46. Jaume I University ePress: Castelló, Spain. HYPERLINK "http://www.e-revistes.uji.es/languagevalue" http://www.e-revistes.uji.es/languagevalue.

DOI: http://dx.doi.org/10.6035/LanguageV.2018.10.3

ISSN 1989-7103

Articles are copyrighted by their respective authors 